\title{
Method for the improvement of mechanical properties of biodegradable polymeric scaffolds
}

\begin{abstract}
At present, a main focus in the field of stent technologies is on the development of biodegradable polymeric stents. Due to inferior material characteristics of polymers compared to permanent metals stents require thicker stents struts to accomplish adequate mechanical properties. Therefore, a thermo-mechanical treatment of polymeric stents was tested, which showed a positive effect on mechanical stent characteristics.
\end{abstract}

Keywords: Biodegradable stents, polymer, thermomechanical treatment, mechanical properties

https://doi.org/10.1515/cdbme-2019-0122

\section{Introduction}

Although current permanent metallic drug-eluting coronary stents show excellent clinical results, the drawbacks of a permanent foreign body in the heart remain. Therefore, a focus in the field of stent technologies is on the development of biodegradable stents as the next step in stent evolution.

Especially degradable thermoplastic polymers used for stent application, e.g. poly(L-lactic acid) (PLLA), show inferior mechanical properties compared to permanent metals. Thus, thicker stent struts are required leading to delayed endothelialization and an increased risk of stent thrombosis. To reduce the specific issues of biodegradable

${ }^{*}$ Corresponding author: Kerstin Schümann: Institute for Biomedical Engineering, Rostock University Medical Center, Friedrich-Barnewitz-Str. 4, 18119 Rostock, Germany, e-mail: kerstin.schuemann@uni-rostock.de

Daniela Arbeiter, Niels Grabow: Institute for Biomedical Engineering, Rostock University Medical Center, FriedrichBarnewitz-Str. 4, 18119 Rostock, Germany

Klaus-Peter Schmitz: Institute for ImplantTechnology and Biomaterials, Institute for Biomedical Engineering, Rostock University Medical Center, Friedrich-Barnewitz-Str. 4, 18119 Rostock, Germany stents a refinement of stent devices with thinner struts in conjunction with adequate mechanical stent properties by advances in material technology is needed.

To achieve a reduced recoil, increased radial strength and enhanced expandability without strut ruptures, a method for thermo-mechanical treatment of polymeric stents was established. This method was tested on stent prototypes with different stent designs and two biodegradable polymeric materials. In vitro tests of mechanical stent properties show the positive impact of thermo-mechanical treatment in comparison to untreated stents.

\section{Materials and methods}

\subsection{Stent designs}

To determine the effect of thermo-mechanical treatment of polymeric stents on their mechanical properties three stent designs were compared. Design 1 and design 2 (Figure 1a, b) consist of interlaced rhombi and differ with regard to their strut lengths. Design 3 (Figure 1c) features double-strut rings. These designs are aimed at providing radial strength in conjunction with high axial bending stiffness.

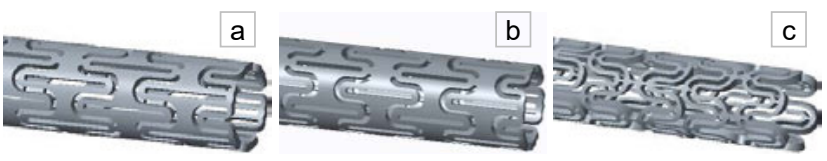

Figure 1: Analyzed stent designs: a) design 1 (interlaced rhombi), b) design 2 (interlaced rhombi with shortened struts), c) design 3 (double-strut rings)

\subsection{Manufacturing of stent prototypes}

Stent prototypes were manufactured by laser cutting from polymeric tubes of PLLA Resomer L214 (Boehringer 
Ingelheim Pharma, Germany) and a polymer blend material of PLLA Resomer L214 and P(4HB) (Tepha, Inc., Cambridge, MA, USA) with a mass ratio of $78 / 22 \%$, according to [1]. Mechanical properties for both materials are summarized in Table 1. The tubes with a nominal inner diameter of $1.4 \mathrm{~mm}$ and a nominal wall thickness of $150 \mu \mathrm{m}$ were fabricated with an automated dip-coating process of stainless steel mandrels. Laser cutting of the different stent designs was performed using a femtosecond laser.

Table 1: Tensile test data of solution cast tubular specimens at $37^{\circ} \mathrm{C}$ of PLLA and PLLA/P(4HB) 78/22 \% (w/w) [1].

\begin{tabular}{llll}
\hline & $\begin{array}{l}\text { Young's } \\
\text { modulus } \\
\text { [GPa] }\end{array}$ & $\begin{array}{l}\text { Tensile } \\
\text { strength } \\
{[\mathrm{MPa}]}\end{array}$ & $\begin{array}{l}\text { Elongation } \\
\text { at break } \\
{[\%]}\end{array}$ \\
\hline PLLA & $2.1 \pm 0.5$ & $45 \pm 2$ & $8 \pm 1$ \\
PLLA/P(4HB) & $1.0 \pm 0.1$ & $36 \pm 3$ & $126 \pm 46$ \\
\hline
\end{tabular}

\subsection{Thermo-mechanical treatment and crimping of stents}

Aim of the thermo-mechanical treatment is the increase of stiffness and strength of the polymer material to improve mechanical stent properties, e.g. small recoil and enhanced radial strength, while retaining uncompromised expandability. The idea of a thermo-mechanical treatment during stent production was shown in $[2,3]$ where a blow molding process with combined radial and axial expansion of polymeric tubes above glass transition temperature $T_{G}$ is described. Accordingly, we established a process for the treatment of polymer stent prototypes.

The laser cut stents were mounted on standard balloon catheters with the nominal stent diameter of $3.0 \mathrm{~mm}$ and suitable according to the stent length and positioned in a water bath at $80^{\circ} \mathrm{C}$. Heating above $\mathrm{T}_{\mathrm{G}}$ (for PLLA: $\mathrm{T}_{\mathrm{G}}=55-$ $65^{\circ} \mathrm{C}$ [4]) causes softening of the polymer and enables molecular movements, e.g. rearrangement or rotation of chain segments. Therefore, orientation of molecular chains along the mechanical load direction is possible. After a holding time to completely heat the stent material the stents were expanded to their nominal diameter by inflating the balloon catheter to nominal pressure. Afterwards, the stents were cooled in air at room temperature.
To set the stent profile for implantation, a subsequent crimping process is required. The stents were crimped with an iris crimping tool in a temperature controlled air chamber slightly below $\mathrm{T}_{\mathrm{G}}\left(50^{\circ} \mathrm{C}\right)$, where a rearrangement of the molecules is inhibited, but the polymer features certain flexibility. Crimping was carried out in multiple diameter steps using an inflated balloon catheter to support the stent structure against collapse.

\subsection{Stent testing}

Stent prototypes were tested regarding their expansion behavior, acute and long-term recoil, as well as radial strength of the expanded stents. Stent testing was performed according to DIN EN ISO 25539-2 using dedicated test procedures described in $[5,6]$.

For stent expansion the balloon was inflated by a computer-controlled pump in a tempered water bath $\left(37^{\circ} \mathrm{C}\right)$. The stent profile during balloon inflation and stent recoil were measured optically with a two-axis laser scanner (ODAC 64 XY, Zumbach Electronic AG, Orpund, Switzerland). The acute recoil was measured immediately after balloon deflation. The long-term recoil was determined after $24 \mathrm{~h}$.

For the measurement of the radial strength the stent prototypes were implanted in thin polyurethane tubes (wall thickness $=0.75 \mathrm{~mm}$ ) fixed into a sealed test chamber. The test chamber was completely filled with temperature controlled water $\left(37^{\circ} \mathrm{C}\right)$ and connected with a computerdriven pressure control unit. The hydraulic pressure in the test chamber was incrementally increased in steps of $2 \mathrm{kPa}$ and applied a radial force to the PUR tube around the stent. The pressure at which the stent loses its support function was noted as the collapse pressure.

\section{Results}

\subsection{Stent prototypes}

Stents with the presented stent designs (see 2.1) were fabricated from PLLA and the PLLA-based blend. Representative photographs of the stents with the different stent designs before and after expansion to their nominal diameter $(3.0 \mathrm{~mm}$ ) are shown in Figure 2. 

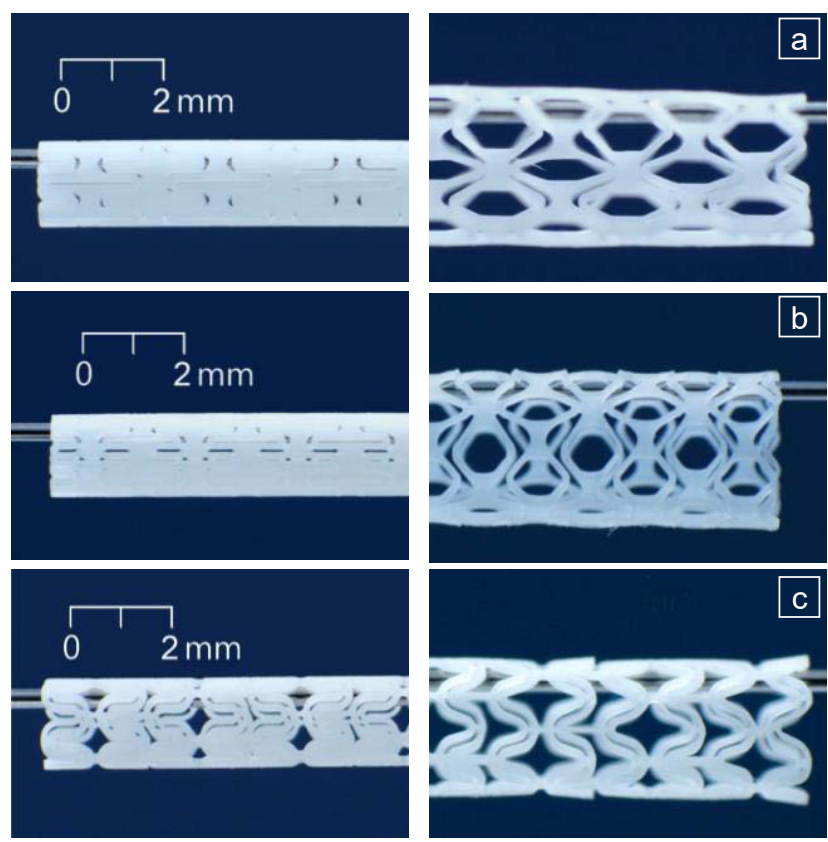

Figure 2: Stent prototypes made of PLLA/P(4HB) 78/22 \% (w/w) without thermo-mechanical treatment before and after expansion to their nominal diameter of $3.0 \mathrm{~mm}$ : a) design 1 (interlaced rhombi), b) design 2 (interlaced rhombi with shortened struts), c) design 3 (double-strut rings)

\subsection{Expansion behavior and recoil}

All of the stents with the different designs made of PLLA/P(4HB) could be expanded without strut ruptures. Because of the limitations of the material behavior, stents with the design 2 (interlaced rhombi with shortened struts) made of PLLA showed strut ruptures during expansion when not subjected to the thermo-mechanical treatment. Therefore, the recoil could not be determined. All other designs, even made of PLLA, and all stents undergoing the thermomechanical treatment could be expanded without strut ruptures.

The results for the acute and long-term recoil after $24 \mathrm{~h}$ for both materials are shown in Figure 3. A distinct difference between acute and long-term recoil becomes obvious which is a result of the viscoelastic material behavior. Due to the thermo-mechanical treatment the acute and particularly long-term recoil can be decreased. Stents made of PLLA show a smaller recoil compared to PLLA/P(4HB) stents. The best results were achieved with design 2. In the treated configuration made of PLLA we obtain an acute recoil of $4.11 \pm 0.04 \%$.
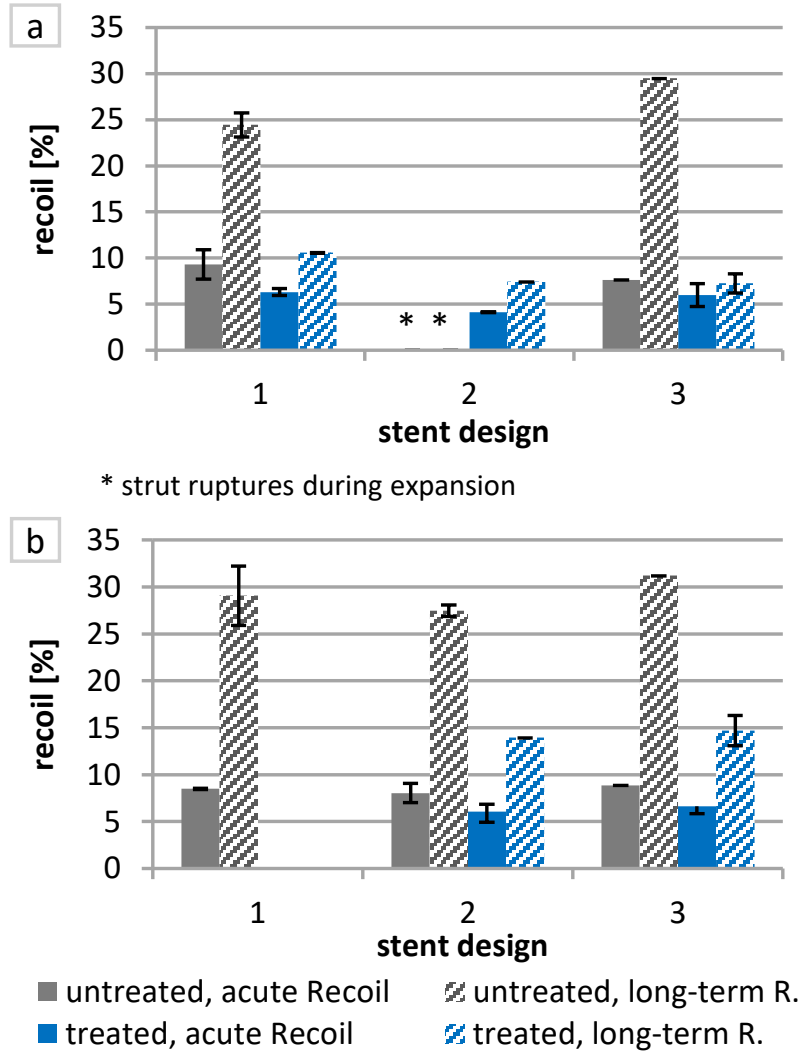

Figure 3: Acute and long-term recoil for the different stent designs with and without thermo-mechanical treatment: a) PLLA, b)

PLLA/P(4HB) 78/22 \% (w/w)

\subsection{Radial strength}

The radial strength of the stents was measured by applying a radial outer pressure. The collapse pressures as determined characteristic value are shown in Figure 4. The collapse pressure of stents made of PLLA is clearly higher than of the PLLA/P(4HB) stents. There is no significant influence on the collapse pressure due to thermo-mechanical treatment for PLLA. For the treated stents made of the blend material the collapse pressure is reduced compared to the untreated stents. The highest value $(86.0 \pm 5.7 \mathrm{kPa})$ was obtained with design 2 made of PLLA subjected to thermo-mechanical treatment.

\section{Discussion}

Prototypes of biodegradable polymeric stents made of PLLA and a blend of PLLA and P(4HB) with a mass ratio of $78 / 22 \%$ featuring different stent designs were manufactured and partially subjected to a thermo-mechanical treatment. 


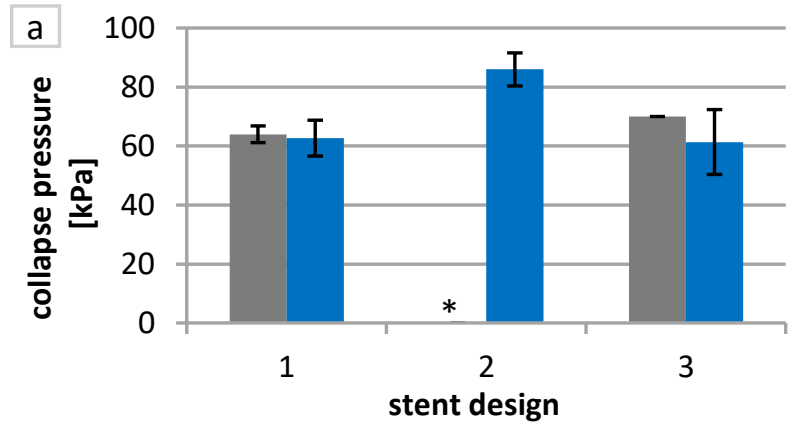

* strut ruptures during expansion

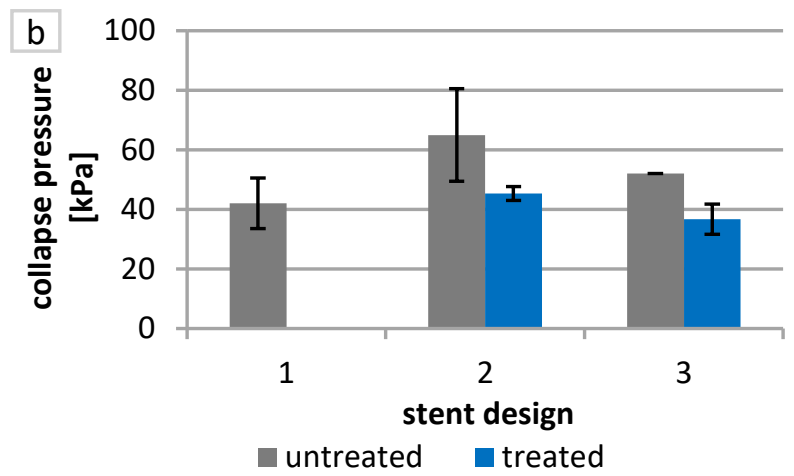

Figure 4: Collapse pressure for the different stent designs with and without thermo-mechanical treatment: a) PLLA, b) PLLA/P(4HB) 78/22 \% (w/w)

Mechanical properties of the stents were determined to examine the influence of the treatment. Stents with design 1 made of the polymer blend and with treatment were not analyzed. Because of strut ruptures no values for recoil and collapse pressure could be determined for stents with design 2 made of PLLA without treatment.

The thermo-mechanical treatment enables smooth expandability of all stent designs without strut ruptures due to prior orientation of the molecular chains according to the expanded state. The results also reveal a notable positive effect of the treatment on the recoil of the stents for both materials regardless of the stent design. This might also be due to the orientation of the molecular chains along the mechanical load directions. For the collapse pressure the treatment shows no significant influence for PLLA and an adversal effect for PLLA/P(4HB). We expected a positive influence on the radial strength. The reason for the obtained results may lie in the complex collapse mechanism of the polymeric stent structure. A plastification of discrete strut cross sections, which is initialized already during expansion, leads to plastic hinges that cause stent collapse. That mechanism probably cannot be hindered by the thermomechanical treatment.

\section{Conclusion}

The conducted thermo-mechanical treatment of polymeric stents is capable to improve expansion behavior and recoil, even for polymer materials with limited ductility. Thermo-mechanical processing is an essential factor to achieve the required mechanical stent properties. Also optimized stent designs and new polymeric materials should be considered. Only if adequate mechanical properties in conjunction with thin stent struts can be accomplished biodegradable polymeric stents have the potential to become an equivalent or even superior alternative to current permanent metallic stents.

\section{Acknowledgment:}

The authors thank the IIB Testing Laboratory "Prüflabor für Kardio+Vaskuläre Produkte" for their support of in vitro testing. Furthermore, we are grateful to Micreon $\mathrm{GmbH}$, Hanover, Germany for laser-cutting of stent prototypes.

\section{Author Statement}

Research funding: This work was partially funded by the Federal Ministry of Education and Research (BMBF) within the project RESPONSE "Partnership for Innovation in Implant Technology". Informed consent is not applicable. Ethical approval: The conducted research is not related to either human or animals use.

\section{References}

[1] Grabow N, Bünger CM, Schultze C, Schmohl K, Martin DP, Williams SF, Sternberg K, Schmitz K-P. A biodegradable slotted tube stent based on poly(L-lactide) and poly(4hydroxybutyrate) for rapid balloon-expansion. Annals of biomedical engineering. 2007;35(12)2031-2038.

[2] Kleiner LW, Tang F. Medical device fabrication process including strain induced crystallization with enhanced crystallization. US 2014/0252675 A1, filed May 15, 2014

[3] Kleiner LW, Oberhauser JP, Glauser T, Wrolstad DK, Wang $Y$. Tube expansion processes and systems for semicrystalline polymers to maximize fracture toughness. US 2011/0278771 A1, filed July 27, 2011

[4] Baird DG, Collias DI. Polymer processing: Principles and design. Hoboken, NJ: Wiley; 2014: 359.

[5] Schmidt W, Behrens P, Behrend D, Schmitz K. Measurement of Mechanical Properties of Coronary Stents according to the European Standard prEN 12006-3. Progress in Biomedical Research. 1999;1(4)45-51.

[6] Schmidt W, Behrens P, Brandt-Wunderlich C, Siewert S, Grabow N, Schmitz K-P: In vitro performance investigation of bioresorbable scaffolds - Standard tests for vascular stents and beyond, Cardiovasc. Revasc. Med. 17(2016) 375-383, DOI: 10.1016/j.carrev.2016.05.001 